\title{
Radio astronomy in the future: impact on relativity
}

\author{
Michael Kramer ${ }^{1,2}$ \\ ${ }^{1}$ MPI für Radioastronomie, Auf dem Hügel 69, 53121 Bonn, Germany \\ email: mkramer@mpifr-bonn.mpg.de \\ ${ }^{2}$ University of Manchester, Jodrell Bank Centre for Astrophysics, Alan-Turing Building, \\ Manchster M13 9PL, UK
}

\begin{abstract}
Radio astronomy has played an important part in the study of relativity. Famous examples include the discovery and exploitation of pulsars for precise binary pulsar tests, the proof of the existence of gravitational radiation and the discovery of the Cosmic Microwave background. In the future, radio astronomy will continue to play a decisive role, assisted by new and upcoming instruments like LOFAR and the SKA. In this review, I will present the revolution that is ongoing in radio astronomical techniques and outline the impact expected on the studies of relativity.
\end{abstract}

Keywords. gravitation, relativity, techniques: radar astronomy, stars: neutron

\section{Introduction}

Astronomical and astrophysical discoveries are usually driven by technological advances that allow us to probe a previously unexplored region of the discovery phase space (e.g. Harwit 1981). This has been clearly demonstrated by the impressive list of fundamental discoveries achieved in radio astronomy (Wilkinson et al. 2004) when a whole new part of the electromagnetic spectrum became available. While we expect similar advances for instance with the anticipated direct detection of gravitational waves using ground-based or space-based detectors, astronomers in other astronomical windows also work towards further technological enhancements. In particular in the electromagnetic spectrum the most notable efforts are clearly those activities towards Extremely Large (optical) Telescopes (ELTs, e.g. Spyromilio 2007) and the Square Kilometre Array (SKA) radio telescope (Schilizzi et al. 2008).

The SKA especially tries to benefit from the recent advances in information technology by replacing expensive metal with flexible and upgradable computing and software solutions. These changes are destined to revolutionize the way we do astronomy: astronomers using the SKA will have to deal with data files of (currently) enormous size, in fact, so large that the data hypercubes may still be several TByte large, even after having been pre-processed on the "grid"; astronomers will be able to observe vast areas of the sky in completely different directions at the same time, and they may even be able to "rewind" time by using a data buffer fed with data from all-sky monitors. The real impact, however, will emerge from the science that will become possible. Simply extrapolating from what we know today, the flood of exciting results should be vast, largely enabled by a huge increase in sensitivity and survey speed. This will clearly have a significant impact on the study of relativity which I try to outline and predict here. 


\section{Radio astronomy: In the past, present and future}

Arguably, most of the fundamental astrophysical discoveries of the last century have been made by radio astronomers. The examples are plenty, among which we find the discovery of the Cosmic Microwave Background (CMB), quasars and radio galaxies, the existence of gravitational lenses, jets and super-luminal motion, dark matter, interstellar molecules and powerful masers and mega-masers. Also, very important was the discovery of pulsars, and the subsequent proof of the existence of gravitational waves and the discovery of the first extra-solar planets. All these discoveries were made after the humble beginnings of Karl Jansky and Grote Reber in the early 1930's, which finally opened up a new part of the electromagnetic spectrum and, hence, allowed astronomers to probe a huge new region of phase space.

The rate of discovery accelerated with the construction of the first "big" dishes, clearly marked with the construction of the Lovell radio-telescope (or the "250-feet" or MkI telescope as it was called at the time) at Jodrell Bank, UK. The current state-of-art still includes the Lovell telescope as the 3rd largest fully-steerable telescope in the world, only trumped in size by the 100-m radio-telescopes at Green Bank, USA, and Effelsberg, Germany. In the Southern hemisphere the largest radio-telescope used by astronomers is still the 64-m dish at Parkes. Special types of telescopes like the 300-m Arecibo telescope (a monolithic dish built into a valley on Puerto Rico and, hence, limited in its ability to track sources), or the Very Large Array (VLA), an interferometer of 27 25-m dishes in New Mexico have played their important part in delivering some of the most exciting results in radio astronomy or astrophysics in general.

Currently, new major telescopes are being constructed, all of which will lead to a new era in radio astronomy. The Atacama Large Millimetre Array (ALMA) is being built in Chile and will vastly improve our sensitivity at very high radio frequencies. The most revolutionary new major facility, however, is perhaps the Low Frequency Array (LOFAR, e.g. van Haarlem 2005). LOFAR will observe at the opposite end of the radio spectrum and will enable studies of the largely unexplored low-frequency radio sky. What makes LOFAR special is not only its large sensitivity at low frequencies, but also the way the telescope works. In contrast to most "classical" radio telescopes, LOFAR has no moving parts but uses antenna elements that receive signals from the whole sky overhead. Combining the signals of the antennae digitally and in software, many independent beams
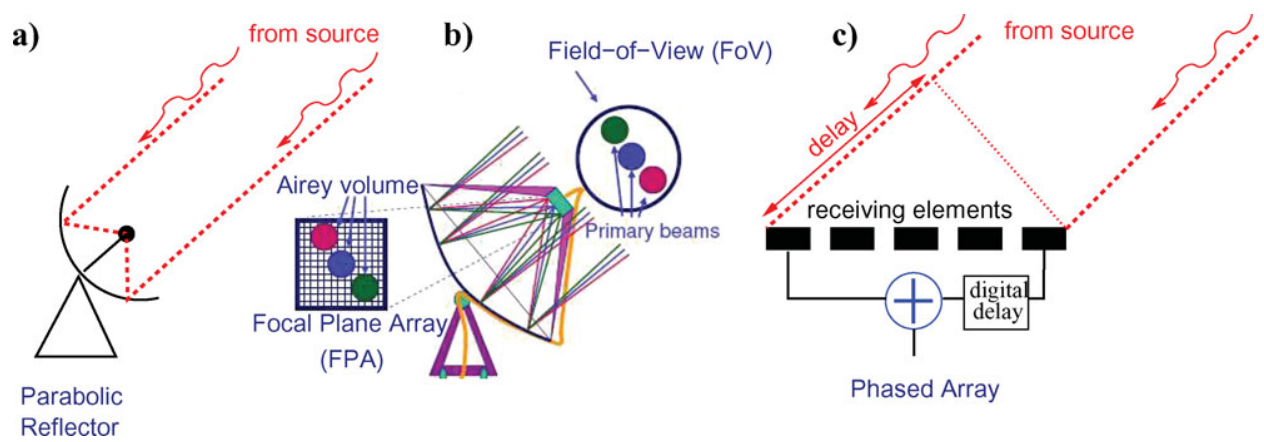

Figure 1. Comparison of pointing a telescope beam towards a source (a) via a conventional parabolic reflector, (b) a parabolic dish equipped with a focal plane array (FPA) or (c) with an aperture array where signals from different receiving elements are combined electronically with appropriate delays to steer a beam to the source direction. Note that in both (b) and (c) the FoV of the telescope is significantly greater than with a conventional telescope. (Figure b courtesy of Andrew Faulkner) 
can be formed that allow the astronomers to observe phenomena and sources in different directions of the sky. In essence, radio astronomers start to replace metal and hardware by computer and software solutions.

LOFAR is an important stepping stone into an era that will not only revolutionize radio astronomy itself, but astronomy and astrophysics - and hence physics - as a whole. The goal of this evolution of our radio eyes is the construction of the largest telescope the Earth has ever seen - the Square Kilometre Array (SKA). I will summarise its main features further below, but it is clear that this enormous step from our current facilities to the gigantic global SKA telescope will require some intermediate steps in order to develop technology and, in particular, affordable techniques and production methods. Therefore, a number of SKA "pathfinder" projects are being undertaken that will explore the technology as well as give a taste of the science that will be enabled by the SKA. Apart from LOFAR, the furthest developed project is the Allen-Telescope-Array (ATA, Welch et al. 2009) in the USA which consists in its initial phase of $426-\mathrm{m}$ dishes, testing the so called large-N-small-D concept. Very significant increases in bandwidths, data transport, data processing and sensitivity are seen in the extensions of MERLIN (the Multi-Element Radio-Linked Interferometer Network) and the VLA to eMERLIN (Garrington 2007) and eVLA (Ulvestad 2007), respectively. In China, a project is underway to build the Fivehundred meter Aperture Spherical Telescope (FAST, Nan 2008) whose 500-m diameter will dwarf even the Arecibo telescope. On the two short-listed SKA sites, two pathfinder telescopes will be completed over the next few years, i.e. MeerKAT in South Africa (Jonas 2007) and ASKAP in Western Australia (Johnston et al. 2008), both of which will have collecting areas of the order of a 100-m dish.

All these exciting new facilities will test aspects of SKA technology but are, excitingly, world-class facilities in their own right and are expected to deliver science (and valuable lessons!) over the next 4-5 years. With this knowledge, construction of the SKA could start around 2013. As an interferometer, the SKA will already be able to deliver science while it is being constructed so that early science with about $10 \%$ of the final collecting area (Phase I) is expected from 2017, while full-SKA operations are anticipated for the early 2020 s.

\subsection{Revolution!}

An important key to achieve the outlined expectations is the increase in telescope sensitivity. For radio astronomy, this is governed by the "radiometer" equation (e.g. Burke \& Smith 2002) which specifies the equivalent flux density for a receiving system of temperature $T_{\text {sys }}$ on a telescope with the equivalent size of $A_{\text {eff }}$ as

$$
S_{\mathrm{min}}=\frac{2 k T_{\mathrm{sys}}}{A_{\mathrm{eff}} \sqrt{\tau \Delta \nu}}=\frac{T_{\mathrm{sys}}}{G} \frac{1}{\sqrt{\tau \Delta \nu}} .
$$

Here, $k$ is the Boltzmann constant, $\tau$ the integration time and $\Delta \nu$ the observing bandwidth. In the last equation, we have defined the telescope gain as $G=A_{\text {eff }} / 2 k$. As most modern receivers are already at the quantum noise limit, the sensitivity cannot be improved further by reducing the system temperature. Hence, an improvement is only possible if we can increase the gain, $G$, (i.e. collecting area), the bandwidth or the integration time. All of this is being addressed by the new telescopes by either increasing the collecting area (e.g. LOFAR, SKA), bandwidth (e.g. eMERLIN, eVLA), or the instantaneous Field-of-View (FoV) which allows one to cover a larger area of sky so that one can integrate longer for the same rate of sky coverage (e.g. ASKAP).

These changes, in particular the increase in bandwidth and FoV, are only possible due to the advances in signal and data processing. Larger bandwidths can be sampled, 
larger data rates can be transferred and larger data volumes can be processed. This is possible using programmable processing units (i.e. Field Programmable Gate Arrays, FPGAs) but also commodity computing power concentrated in "Beowulf Clusters" and even increasingly due to the use of game console hardware, so called GPUs. In particular the latter shows that radio astronomers try to utilise technical developments that are driven outside science but in consumer electronics or telecommunication. These advances indeed allow astronomers to observe large fractions of the sky at once using so-called "phased arrays" where receiving elements are sensitive to vastly different directions or even the whole sky. Their signals are added in hard- or software using particular delays to achieve directionality (see Figure 1). The ultimate number of beams is only limited by the available computing power.

Phased arrays laid flat on the ground are also called "aperture arrays" (AAs), and they are indeed sensitive to the whole sky overhead, although the effective area drops as the cosine of the zenith angle. AAs are an essential part of the European plans to build the SKA. Phased arrays can also be installed in the prime focus of conventional dish telescopes in which case they are referred to as "focal plane arrays" (FPAs). An FPA allows one to detect light rays via paths that are inclined to the geometrical axis of the telescope (see Figure 1). The result is a significant enlargement of the telescope's FoV, sometimes up to an order of magnitude or more. With the combination of the signals from different FPA elements, the enlarged FoV can be fully filled. First examples of both AA and FPA type are very promising, while the remaining technological challenges lie in achieving low system temperatures and in the required data handling and processing.

\subsection{What is it? A telescope, a supercomputer? Both!}

The described efforts to increase the effective sensitivity of new radio telescopes all have one feature in common: they all require an early digitization of the signals and a very significant increase in the processing requirements. This goes in hand with an increase in the number of bits used for digitization in order to be more resilient to man-made radio interference signals. Moreover, a significant increase in collecting area is hard or impossible to achieve with a single monolithic structure, but most new radio telescopes are built as interferometers, i.e. an array of small telescopes that are combined to synthesize a filled aperture. For such instruments, the spatial resolution is given by the largest "baseline", $b_{\max }$, i.e. the largest distance between a pair of telescopes. Small "pencil beams" are then synthesized to fill the full FoV of the telescope which in case of single dish elements is as large as the FoV of a single element ("primary beam") or of different size in case of AAs and their combination. Forming an astronomical image with an interferometer is done by combining the signals of the array elements using a correlator. As the signal of each telescope needs to be combined or correlated with every other element, the processing requirements increase with the square of number of antennas, the cube of baseline length and the FoV solid angle.

The actual configuration of the telescope, such as type, size and number of receiving elements, their relative spacing and the observing bandwidth and frequency, will be the result of a complex optimization process regarding costs, technical feasibility and science drivers, but in order to fill a FoV of a few square degree one needs $N_{\text {pix }} \sim\left(b_{\max } / D\right)^{2} \sim$ $10^{4}-10^{9}$ pixels and about $N_{\text {ops }} \sim 10^{15}$ operations per second for a primary beam of a telescope of diameter $D$. In addition, the obtained data need to be processed on-the-fly, as data rates of several TByte per second as estimated for the SKA cannot be transferred over large distances. All in all, these estimates put a telescope like the SKA safely in the region of the most powerful Exaflop supercomputers anticipated for the next decade. 


\subsection{The Square-Kilometre Array (SKA)}

Essentially all upcoming and future telescopes will contribute in one way or the other to advances in the field of relativity. LOFAR will find a large number of neutron stars that are potential sources for tests of relativity or pulsar timing array experiments that attempt to directly detect gravitational waves Stappers et al. (2007). Even though these experiments will be performed at high frequencies, LOFAR can find appropriate sources and also monitor the interstellar weather that needs to be corrected for in high precision timing (see contribution by Matthew Bailes). FAST will have a collecting area that allows us to find and time pulsars that will be significantly better than currently achieved with Arecibo (Smits et al. 2009). The eVLA promises to give us a first good glance at the pulsar population orbiting the super-massive black hole in the Galactic Centre. However, the real big advance in studies of gravitational theories will be achieved with the SKA, so that we concentrate on this science instrument.

The exact configuration of the SKA will be determined after the ongoing global R\&Dphase is completed and a SKA design is presented in 2012. The current efforts for this project that includes 19 countries at the time of writing cover all possible areas, ranging from developing receiving elements to amplifiers, beam-formers, software and governance structure and much more. The results will be compared to the current reference design (Schilizzi 2007) which is outlined in the following.

The defining feature of the SKA will be overwhelming sensitivity due to enormous collecting area. As the name suggests, the total collecting area for the SKA is planned to be one square-kilometer. However, for practical purposes it makes sense to define the sensitivity of the SKA in terms of collecting area and system temperature, as $S_{\mathrm{min}}$ directly depends on them (see Eqn. 2.1). The target sensitivity is $2 \times 10^{4} \mathrm{~m}^{2} \mathrm{~K}^{-1}$ which we will refer to as one SKA unit. The SKA is likely to consist of a sparse AA of tiled dipoles in the frequency range of 70 to $500 \mathrm{MHz}$, and above $500 \mathrm{MHz}$ the following three implementations are considered:

A) $300015-\mathrm{m}$ dishes with a single pixel feed, a sensitivity of $0.6 \mathrm{SKA}$ units, $T_{\text {sys }}=30 \mathrm{~K}$ and $70 \%$ efficiency covering the frequency range of $500 \mathrm{MHz}$ to $10 \mathrm{GHz}$.

B) $200015-\mathrm{m}$ dishes with FPAs from $500 \mathrm{MHz}$ to $1.5 \mathrm{GHz}$, a sensitivity of $0.35 \mathrm{SKA}$ units, a FoV of $20 \mathrm{deg}^{2}, T_{\text {sys }}=35 \mathrm{~K}$ and $70 \%$ efficiency and a single pixel feed from 1.5 to $10 \mathrm{GHz}$, with $T_{\text {sys }}=30 \mathrm{~K}$.

C) A combination of dense AAs with a FoV of $250 \mathrm{deg}^{2}$, a sensitivity of 0.5 SKA units, covering the frequency range of 500 to $800 \mathrm{MHz}$ and $240015-\mathrm{m}$ dishes with a single pixel feed covering the frequency range of $800 \mathrm{MHz}$ to $10 \mathrm{GHz}$, a sensitivity of $0.5 \mathrm{SKA}$ units, $T_{\text {sys }}=30 \mathrm{~K}$ and $70 \%$ efficiency.

The signals of these receiving elements can be combined to form independent FoVs.

Most of the collecting area will be concentrated in a central core of the SKA, i.e. $20 \%$ of the collecting area will be located within a $1 \mathrm{~km}$ radius and $50 \%$ within a $5 \mathrm{~km}$ radius. The remainder of the receiving elements will be distributed in stations along, probably, log-spiral arms with baselines extending to $3000 \mathrm{~km}$ distance in order to enable also high resolution imaging and high precision astrometry. Further resolution will be available via intercontinental baselines between Australia and Southern Africa.

The SKA will be built in three phases. In Phase I about 0.1 SKA unit will be available in the core region covering the low and mid frequencies by 2017. In Phase II, the full array will be completed by 2022. Finally, in Phase III high frequency capability for observations potentially up to $40 \mathrm{GHz}$ will be added from 2022 .

The SKA will be a flexible, multi-purpose observatory that will be able to serve the whole astronomy community as the premier imaging and surveying instrument. The 


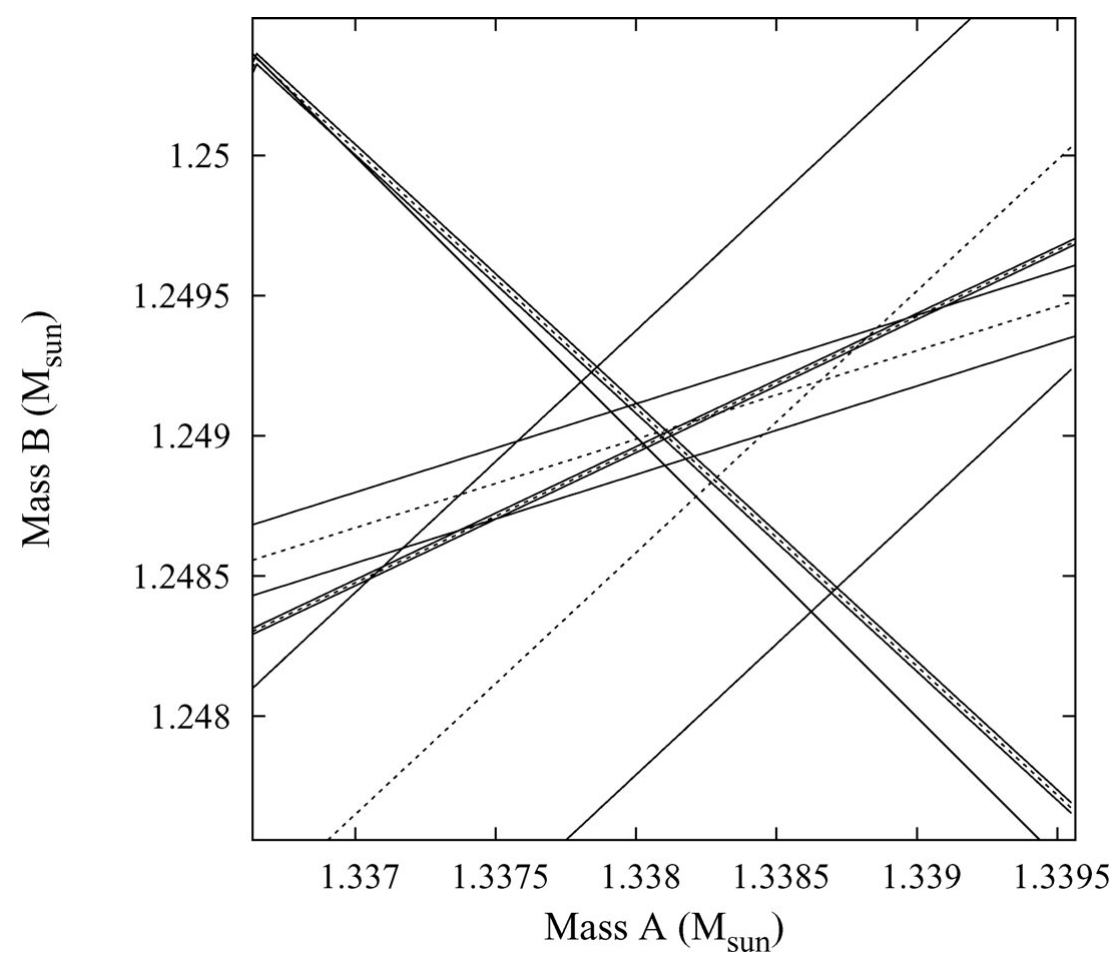

Figure 2. Mass-mass diagram for the Double Pulsar in 20 years from now, based on simulated TOAs (see Kramer \& Wex 2009 for details). The solid lines represent the two-sigma errors of the post-Keplerian parameters $\left(\dot{\omega}, \gamma, s, \dot{P}_{\mathrm{b}}\right)$ and the mass ratio $\left(R \equiv m_{\mathrm{A}} / m_{\mathrm{B}}\right)$. The $\dot{\omega}$ line was assuming a vanishing moment-of-inertia for pulsar A. Hence, the contribution of the spin-orbit coupling to the total precession of periastron, $\dot{\omega}_{\mathrm{SO}}$, can be read off the offset between the $\dot{\omega}$-line and the point of intersection of the $\dot{P}_{\mathrm{b}}$ and the $s$ curves. As the masses of pulsar A and B are roughly equal, the slopes of the $\dot{\omega}$ and the $\dot{P}_{\mathrm{b}}$ curve are roughly the same close to the $P_{\mathrm{b}}-s$ intersection. Hence, the determination of $\dot{\omega}_{S O}$ is much less sensitive to errors in $s$ than to errors in $\dot{P}_{\mathrm{b}}$.

design will ensure that it will be a flexible instrument rather than a fine-tuned experiment, even though five Key Science Projects (KSPs) have been selected by the international community. These KSPs drive essential parts of the design in order to ensure that all current science goals can be achieved. Still, versatility is a key element in the construction of the SKA which also includes natural upgrade paths: Eventually the SKA's power will only be constrained by the available computing power, so that the estimated operation costs budget includes items to constantly replace and upgrade SKA processing capabilities.

The KSPs cover a wide area of topics from fundamental physics to general astrophysics and cosmology. These are summarized in the SKA Science Book edited by Carilli \& Rawlings (2004) as Extreme tests of general relativity (Kramer et al. 2004, see also below), Galaxies, cosmology, dark matter and dark energy (Rawlings et al. 2004), . Probing the dark ages the first black holes and stars (Carilli et al. 2004), The origin and evolution of cosmic magnetism (Gaensler et al. 2004), and the Cradle of life (Lazio et al. 2004). The versatility of the SKA will also allow a huge range of (astro-)physics to be conducted in addition to the these KSP. This includes obviously the likely possibility to discover unknown phenomena and physics (Wilkinson et al. 2004). 


\section{Applications in Relativity}

It is clear that the SKA will have a huge impact on the study of relativity. Observations of weak lensing, the cosmic web, and baryonic oscillations via a billion galaxy survey will firmly establish the nature of dark energy. Also, spectroscopy of highly redshifted quasers will probe the possible variation of fundamental constants, while the monitoring of water mega-masers allow us to probe the expansion of the local Universe. Most importantly, however, will be the search for and timing of radio pulsars. With SKA sensitivity one can essentially find all active Galactic pulsars beaming towards Earth, providing a "Galactic Census" of pulsars (Cordes et al. 2004). We expect to find about 20,000 to 30,000 pulsars including 1,000 millisecond pulsars and 100 relativistic binaries (Smits et al. 2009).

\subsection{Astrometry E Binary Pulsar Tests}

Astrometry and in particular pulsar timing of millisecond pulsars should allow us to measure proper motions with a precision of about 100 nas/yr and parallaxes of the order of 10-20 $\mu$ as, i.e. distances of up to $50-100 \mathrm{kpc}$. Astrometric measurements are not only useful to potentially trace the pulsars' movement in the gravitational field of the Galaxy or globular clusters, but parallax measurements are also needed to correct for relative acceleration between the pulsar and the solar system barycentre which affects the observed period derivatives (for spin and orbit) of relativistic binaries (e.g. Lorimer \& Kramer 2005 and contributions by Bailes and Stairs). This is essential if radiative aspects of a theory of gravity are to be tested. The prospects for tests when using the SKA to study the Double Pulsar (Lyne et al. 2004) are discussed in detail by Kramer \& Wex (2009). Essentially, with the SKA we expect an improvement in timing precision by a factor of $\sim 100$, leading to precision tests of general relativity that even exceed the precise tests done in the solar system's weak field regime today. (For a detailed description of tests with pulsars see Ingrid Stairs' contribution.) Moreover, at the same time, the Gaia satellite mission is expected to significantly improve our knowledge of the galactic potential (Brown 2008), and hence lead to a much better understanding of the galactic contributions to the observed period derivative. All this will allow us to measure the moment-of-inertia of pulsar A in the Double Pulsar system (see Figure 2). Furthermore, we expect to measure the previously unmeasured relativistic deformation of the orbit, providing an additional test for general relativity and other theories of gravity (Kramer \& Wex 2009). Unfortunately, FAST will not be able to see the Double Pulsar but improvements in timing this system could already be achieved with the Large European Array for Pulsars (LEAP, Kramer priv. comm.). In LEAP the major European telescope are added together to form the equivalent of an illuminated Arecibo dish, increasing the timing precision accordingly.

\subsection{Alternative theories 6 Black hole properties}

What makes a binary pulsar with a black-hole companion so interesting is that it has the potential of providing a superb new probe of relativistic gravity. As pointed out by Damour \& Esposito-Farese (1998), the discriminating power of this probe might supersede all its present and foreseeable competitors. The reason lies in the fact that such a system would be very sensitive to strong gravitational self-field effects, making it for instance an excellent probe for tensor-scalar theories. Moreover, Wex \& Kopeikin (1999) showed that the measurement of classical and relativistic spin-orbit coupling in a pulsar-black hole binary, in principle, allows us to determine the spin and the quadrupole moment of the black hole. This would test the "cosmic censorship conjecture" and the "no-hair 
theorem". While Wex \& Kopeikin (1999) pointed out that with current telescopes such an experiment would be almost impossible to perform (with the possible exception of pulsars about the Galactic center black hole), Kramer et al. (2004) demonstrated that the SKA sensitivity should be sufficient. Indeed, this experiment benefits from the SKA sensitivity in multiple ways. On one hand, it provides the required timing precision but it also allows to perform the Galactic Census which should eventually deliver the sample of pulsars with a black hole companion.

\subsection{Gravitational wave astronomy}

While pulsars already provide the indirect evidence for the existence of gravitational waves (GW), they can also be used to detect and study them directly, since the timing residuals are affected by a passing GW wave as each pulsar and the Earth can be considered as free masses, whose positions respond to changes in the space-time metric (e.g., Sazhin 1978, Detweiler 1979). An update on the current efforts and details of such experiments is given in the contribution by Hobbs. For our purposes it is important to note that a perturbation of the pulsar and Earth position by a GW would lead to timing residuals of the order of $\sigma \sim h_{c}(f) \times T^{1.5}$ where $h_{c}(f)$ describes the characteristic amplitude of the GW per unit logarithmic interval of frequency, and $T$ is the total observing time. Importantly, the sensitivity of GW detection using pulsars scales directly with the achieved timing precision.

Despite the apparent simplicity of the experiment, the timing precision required for the detection of GWs is very much at the limit of what is technically possible today. Only a stochastic background (rather than a single GW source) is likely to be detected today, as it would produce a correlated quadrupole signature among a network or "array" of timed millisecond pulsars on the sky. This facilitates the recognition of a signal originating, for instance, from the orbital motion of binary super-massive black holes in the early Universe (see Hobbs' contribution for more detail). A variety of sources is expected to emit GWs in the nHz-frequency range detectable by a "pulsar timing array" (PTA). Such PTA experiments and, in particular LEAP, harvesting the combined collecting power of Europe's largest telescopes, will have a good chance of detecting GWs, but only with the SKA can a detection be guaranteed and the properties of GWs (e.g. spectrum of a stochastic background, polarisation) be studied. Due to the increased timing precision achievable with the SKA and a large number of suitable sources discovered, a PTA with the SKA would achieve a sensitivity that even exceeds that of LISA (Kramer et al. 2004).

Different types of signals can be detected and studied with the SKA, i.e. stochastic, burst, and periodic signals. A stochastic gravitational wave background should arise from a variety of sources. Cosmological sources include inflation, string cosmology, cosmic strings and phase transitions (Kramer et al. 2004). The expected correlation signal among the PTA pulsars can be measured with very high precision, so that the polarisation properties of GW can be probed and compared with the predictions of GR (Lee et al. 2008). The SKA will also detect single sources of GW emission, such as the periodic GW signal of a binary super-massive black hole in the centre of a nearby Galaxy (e.g. Jenet et al. 2004) Here, the SKA and LISA will be complementary: On one hand, both instruments can see similar super-massive binary black hole systems but at different evolutionary stages, i.e. LISA will pick up SKA sources close to their merger. On the other hand, the SKA can observe GW emission of systems that are typically more massive than the LISA sources. We should also be able to discover compact pulsar binaries that emit in radio simultaneously with continuous GW emission detectable with LISA. 
Moreover, the black holes studied with the SKA (stellar and the supermassive black hole in the Galactic Centre, see Section 3.2) will be complementary and partly overlapping with the population of black holes to be studied with Advanced LIGO and LISA.

Besides these guaranteed GW signals, the SKA should also be able to measure burst emission from, for instance, explosive events. In the GW window, pulsar glitches may also produce observable events, where the SKA would help to pinpoint the exact time of such an event. Fundamental properties of gravitation can also be tested: if GWs are governed by a massive field propagation law, GWs with frequency less than $m_{\mathrm{g}} c^{2} / h$ cannot propagate. Hence detecting GWs with a 5 to 15 year period (ie. the frequency range covered by PTAs) reduces the upper bound on gravitation mass $m_{\mathrm{g}}$ by a factor of 15 to 50 (F. Jenet, priv. comm.)

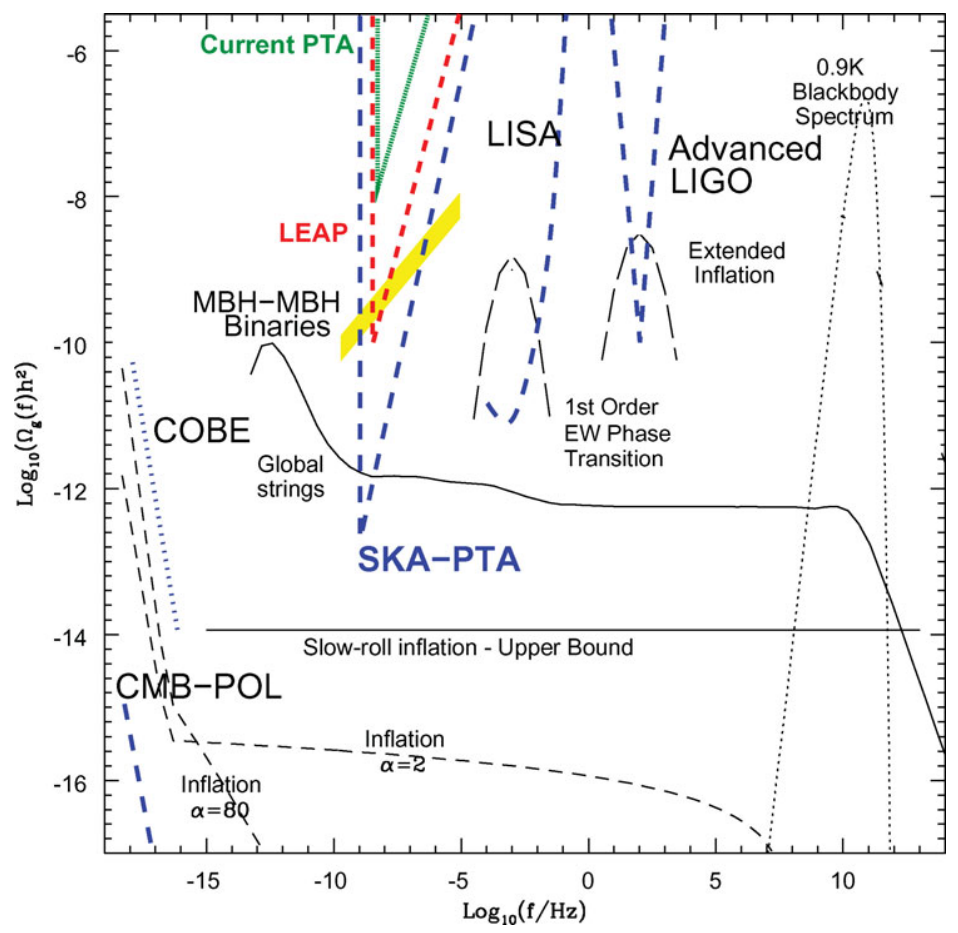

Figure 3. Summary of the potential cosmological sources of a stochastic gravitational background, including inflationary models, first-order phase transitions and cosmic strings and a primordial 0.9K black-body gravitation spectrum as presented by Battye \& Shellard (1997). We also overlay bounds from COBE, from current millisecond pulsar timing and the goals from CMB polarization, LISA and Advanced LIGO. The PTA provided by the SKA will improve on the current MSP limit by about four orders of magnitudes. The gray area indicates the spectrum of an additional astrophysical background caused by the merger of massive black holes (MBHs) in early galaxy formation (see text). For this background, $\Omega_{g w} \propto f^{2 / 3}$, whilst its amplitude depends on the $\mathrm{MBH}$ mass function and merger rate. The uncertainty is indicated by the size of the shaded area. The expected sensitivities for LEAP and SKA are marked.

\section{Conclusions}

The past has shown that large astronomical progress is achieved when new phase space is probed by advances in technology. Radio astronomy, in particular, has benefited greatly 
from such advances and will continue to do so in the future. In fact, we are experiencing a revolution in the way radio astronomy is conducted as our instruments allow us now to directly "digitize" our photons. This has enormous consequences, since we can greatly benefit from the continuing advances in digital electronics, telecommunication and computing. The results are jumps in achievable bandwidths, FoVs, frequency coverage and also collecting area. The global efforts will culminate in the construction of the SKA as the world's largest and most powerful telescope. Importantly, the SKA, as a versatile observatory, will revolutionize many areas of astrophysics and fundamental physics.

\section{References}

Battye, R. A. \& Shellard, E. P. S. 1996, Class. Quantum Grav., 13, A239-A246

Brown, A. G. A., in American Institute of Physics Conference Series, Bailer-Jones (ed), Learning about Galactic structure with Gaia astrometry, American Institute of Physics Conference Series, 1082, 209

Burke, B. F. \& Smith, F. G. 2002, An introduction to Radio Astronomy, (Cabridge: Cambridge University Press)

Carilli, C. L. \& Rawlings, S. 2004, New Astronomy Review, 48, 979

Carilli, C. L., Furlanetto, S., Briggs, F., Jarvis, M., Rawlings, S., \& Falcke, H. 2004, New Astronomy Review, 48, 1029

Cordes, J. M., Kramer, M., Lazio, T. J. W., Stappers, B. W., Backer, D. C., \& Johnston, S. 2004, New Astronomy Review, 48, 1413

Damour, T. \& Esposito-Farèse, G., Phys. Rev. D, 1998, 58(042001), 1

Detweiler, S. 1979, ApJ, 234, 1100

Gaensler, B. M., Beck, R., \& Feretti, L. 2004, New Astronomy Review, 48, 1003

Garrington, S. T., 2007, in From Planets to Dark Energy: the Modern Radio Universe, eMERLIN, The University of Manchester, UK. Published online at SISSA, Proceedings of Science, p.9

Harwit, M., 1981, Cosmic discovery. The search, scope, and heritage of astronomy, Brighton: Harvester Press, 1981

Jenet, F. A., Lommen, A., Larson, S. L., \& Wen, L. 2004, ApJ, 606, 799

Johnston, S. et al., 2008, Experimental Astronomy, 22, 151

Jonas, J. 2007, in From Planets to Dark Energy: the Modern Radio Universe, The University of Manchester, UK. Published online at SISSA, Proceedings of Science, p.7

Kramer, M. \& Wex, N. 2009, Class. Quant. Grav., 26(7), 073001

Kramer, M., Backer, D. C., Cordes, J. M., Lazio, T. J. W., Stappers, B. W., \& Johnston, S. 2004, New Astronomy Review, 48, 993

Lazio, T. J. W., Tarter, J. C., \& Wilner, D. J. 2004, New Astronomy Review, 48, 985

Lee, K. J., Jenet, F. A., \& Price, R. H. 2008, ApJ, 685, 1304

Lorimer, D. R. \& Kramer, M. 2005, Handbook of Pulsar Astronomy, (Cambridge: Cambridge University Press)

Lyne, A. G. et al., 2004, Science, 303, 1153

Nan, R. 2008, in Society of Photo-Optical Instrumentation Engineers (SPIE) Conference Series, Introduction to FAST: five hundred meter Aperture Spherical radio Telescope, 7012

Rawlings, S., Abdalla, F. B., Bridle, S. L., Blake, C. A., Baugh, C. M., Greenhill, L. J., \& van der Hulst, J. M. 2004, New Astronomy Review, 48, 1013

Sazhin, M. V. 1978, Sov. Ast., 22, 36

Schilizzi, R. T., Dewdney, P. E. F., \& Lazio, T. J. W. 2008, in Society of Photo-Optical Instrumentation Engineers (SPIE) Conference Series, The Square Kilometre Array, 7012

Schilizzi, R. 2007, in From Planets to Dark Energy: the Modern Radio Universe, The Square Kilometre Array, The University of Manchester, UK. Published online at SISSA, Proceedings of Science, p.2

Smits, R., Lorimer, D. R., Kramer, M., et al., 2009, A\&A, in press 
Smits, R., Kramer, M., Stappers, B., et al., 2009, A\&A, 493, 1161

Spyromilio, J. 2007, in From Planets to Dark Energy: the Modern Radio Universe, Extremely Large Telescopes, The University of Manchester, UK. Published online at SISSA, Proceedings of Science, p.3

Stappers, B. W., van Leeuwen, A. G. J., Kramer, M., Stinebring, D., \& Hessels, J. 2007, arXiv:astro-ph/0701229

Ulvestad, J. 2007, in From Planets to Dark Energy: the Modern Radio Universe. The EVLA as a scientific and technical pathfinder for the SKA, The University of Manchester, UK. Published online at SISSA, Proceedings of Science, p. 8

van Haarlem, M. P. 2005, in Gurvits L. I., Frey S., Rawlings S. (eds), LOFAR: The Low Frequency Array", EAS Publications Series, 15, 431

Welch, J. et al., 2009, arXiv:astro-ph/0904.0762

Wex, N.\& Kopeikin, S. 1999, ApJ, 513, 388

Wilkinson, P. N., Kellermann, K. I., Ekers, R. D., Cordes, J. M., \& Lazio, T. J. W. 2004, New Astronomy Review, 48, 1551 